\title{
Como se tornar um pai presente: impactos de um grupo de pais ${ }^{1}$
}

\author{
How to become a present father: impacts of a group of parents
}

Fabiana Cia ${ }^{[0]}$, Elizabeth Joan Barham ${ }^{[b]}$

\begin{abstract}
${ }^{[a]}$ Doutora em Educação Especial, professora adjunta do Departamento de Psicologia e do Programa de Pós-Graduação em Educação Especial da Universidade Federal de São Carlos (UFSCar), São Carlos, SP - Brasil, e-mail: fabianacia@ hotmail.com)

${ }^{\left[{ }^{[b]}\right.}$ Professora adjunta do Programa de Pós-Graduação em Psicologia e do Departamento de Psicologia da Universidade Federal de São Carlos (UFSCar), São Carlos, SP Brasil, e-mail: lisa@ufscar.br
\end{abstract}

Recebido: $14 / 02 / 2012$ Received: 02/14/2012

Aprovado: $11 / 06 / 2012$ Approved: 06/11/2012

\begin{abstract}
Resumo
Este estudo avaliou a eficácia de um programa de intervenção para pais sobre o envolvimento paterno. Os participantes foram divididos em três grupos. No grupo experimental 1 (GE1), formado por 29 pessoas, (pais, mães e crianças), apenas os pais receberam intervenção. No grupo experimental 2 (GE2), formado por 36 pais e mães e 38 crianças, apenas as mães receberam intervenção. No grupo controle, composto por 34 pessoas (pais, mães e crianças), pais e mães não receberam intervenção. Para avaliar a frequência do envolvimento paterno, pais, mães e crianças de todos os grupos responderam ao questionário "Avaliação do relacionamento pai-filho" em três momentos diferentes: antes da intervenção (pré-teste), após a intervenção (pós-teste) e após um ano do início da intervenção (follow-up). Nos grupos GE1 e GE2, as frequências de comunicação entre pai e filho e de participação do pai nas atividades escolares, culturais e de lazer do filho foram estatisticamente maiores no pós-teste do que no pré-teste.
\end{abstract}

Palavras-chave: Envolvimento paterno. Estudos de intervenção. Fase de Transição.

\begin{abstract}
This study assessed the effectiveness of an intervention program for parents on parental involvement. Participants were divided into three groups. In experimental group 1 (GE1), composed of 29 people (parents and children), only fathers received intervention. In experimental group 2 (GE2), composed of 36 parents and 38 children, only mothers received intervention. In control group, composed of 34 people (parents and children), parents received no intervention. To assess the frequency of father involvement, parents and children of all groups answered the questionnaire "Assessment of father-child relationship" in three different moments: before the intervention (pre-test), after the intervention (post-test) and one year after the beginning of the intervention (follow-up). In groups GE1 and GE2, the frequencies of communication between father and child and of involvement of the father in school, cultural and leisure activities of the child were significantly higher in the post-test than in the pre-test
\end{abstract}

Keywords: Father involvement. Intervention studies. Transition phase.

${ }^{1}$ Apoio Financeiro: Fapesp. Este trabalho é derivado da Tese de Doutorado da primeira autora, no Programa de Pós-Graduação em Educação Especial, da Universidade Federal de São Carlos.

Psicol. Argum. 2014 jan./mar., 32(76), 139-150 


\section{Introducão}

O conceito de paternidade tem experimentado drásticas mudanças decorrentes das modificações econômicas, sociais e culturais que a família vem sofrendo ao longo do tempo. Entre a década de 1930 e meados da década de 1980, a maior parte dos pais desempenhava suas tarefas educativas baseado na tradicional divisão de papéis, segundo o gênero (Biasoli-Alves, Caldana \& Silva, 1997). Com a revolução feminista, a partir da década de 1970, a porcentagem de mulheres exercendo atividades remuneradas vem progressivamente aumentando (Raley, Mattlingly \& Bianchei, 2006). 0 envolvimento da mulher no mercado de trabalho tem favorecido economicamente a família, mas essa maior equidade econômica gerou transformações nos papéis atribuídos ao gênero, tanto no ambiente profissional quanto no familiar (Brandth \& Kvande, 2002; Dantas, Jablonski \& Féres-Carneiro, 2004).

Apesar de os homens dos tempos atuais estarem mais envolvidos na educação e nos cuidados diários com seus filhos do que nas gerações anteriores, as diferenças entre os gêneros persistem, com as mulheres ainda sendo as principais responsáveis por estas tarefas, mesmo exercendo alguma atividade remunerada (como trabalho autônomo ou assalariado com carga horária de até 40 horas semanais), e os homens passando maior número de horas semanais em alguma atividade remunerada (Ahmed \& Bould, 2004; Raley et al., 2006).

Então, verifica-se que, com o tempo, os homens começaram a se envolver mais nos cuidados e nas atividades com os filhos. Assim, os pesquisadores sociais passaram a enfatizar a importância de estudar a interação entre pai e filho. A partir desta nova geração de trabalhos, surgiu o reconhecimento de que os pais desempenham papéis complexos (multidimensionais) e que muitos aspectos de sua influência são indiretos (Lamb, 1997).

Observa-se que essas mudanças nos papéis de gênero não ocorrem com a mesma frequência e intensidade em todas as famílias. 0 que se encontra hoje são famílias com diferentes maneiras de fazer a divisão das suas tarefas. Coexistem modelos familiares diversos: em alguns é seguida a tradicional divisão de papéis, em outros, maridos e esposas dividem melhor as tarefas domésticas e educativas, e há também famílias nas quais as mulheres são as principais mantenedoras financeiras do lar (Fleck \& Wagner, 2003; Rocha-Coutinho, 2003).

Embora a maior parte dos pais e mães ainda se envolva em atividades diferentes ou interaja com seus filhos de forma diferente (por exemplo, o comportamento dos pais e das mães ao brincarem com seus filhos são diferentes, mesmo que ambos estejam participando da mesma brincadeira), é importante enfatizar que ambos desempenham papéis importantes para todos os aspectos do desenvolvimento infantil (Guille, 2004; Lamb, 1997). Por exemplo, várias pesquisas apontam que os pais se ocupam mais em proporcionar lazer fora de casa aos filhos, enquanto as mães são mais focadas nas atividades da vida diária. Nota-se uma nova divisão razoavelmente estruturada de atividades, com os pais se ocupando mais da parte social (extra-familiar) e de lazer, e as mães dando cuidados diários aos filhos em casa e mantendo as relações familiares extensas (Cia \& Barham, 2005; Cia, Pereira, Del Prette \& Del Prette, 2006; Hawkins, Amato \& King, 2006).

Conclui-se que apesar de haver transformações nos papéis masculinos e femininos, ainda há um menor envolvimento dos homens nas interações, nos cuidados diários e na realização de atividades com os filhos, pois este envolvimento paterno pode ser afetado por vários fatores como os mencionados anteriormente. No entanto, passa-se por um momento histórico, quando o envolvimento materno está se modificando e tornando o envolvimento paterno mais importante para manter investimentos educativos por parte da família. Diante disso, fica evidente a importância de buscar maneiras de encorajar o envolvimento paterno a superar estas barreiras, uma vez que, em muitas famílias, os homens podem estabelecer pouco envolvimento com os filhos (Cia \& Barham, 2005; Cia, Pamplin \& Del Prette, 2006; Flouri \& Buchanan, 2003).

\section{Programas de intervenção com pais}

McMahon (1999) define o treinamento de pais como um enfoque para o tratamento dos problemas infantis, capacitando os pais a modificar o comportamento de seus filhos em casa. Os pais se reúnem com um terapeuta ou treinador que os ensina a usar uma série de procedimentos específicos para modificar sua interação com os filhos, para auxiliar o comportamento pró-social e diminuir o 
comportamento desviado. 0 treinamento de pais tem recebido uma notável atenção nas últimas décadas e tem sido empregado principalmente no tratamento de crianças que mostram problemas de comportamento manifestos, como birras, agressão e desobediência excessiva. Segundo McMahon, programas de intervenção com pais devem ter os seguintes aspectos: (a) o tratamento se realiza principalmente com os pais e não com o foco na criança; (b) o conteúdo desses programas inclui instruções dos princípios de aprendizagem social, treinamento na definição, vigilância e seguimento do comportamento da criança, procedimentos de reforço positivo, de extinção e punição leve (como ignorar) e o treinamento de dar instruções e ordens claras; (c) uso de instruções, modelação, representação de papéis e ensaio comportamental.

Para Freitas (2005), os programas direcionam os pais a ser mediadores do tratamento do filho, pois eles se tornam motivados a investir nos problemas socioemocionais dos filhos porque o microssistema familiar está dentro do sistema social mais amplo. Assim, os mesmos comportamentos inadequados da criança que podem ser mantidos no sistema familiar, podem ser punidos em outro sistema social, como na escola ou na comunidade, estabelecendo contingências para os pais procurarem modificar os comportamentos inadequados das crianças.

Como um exemplo de intervenção para pais, Bolsoni-Silva, Salina-Brandão, Rosin-Pinola e Versuti-Stoque (2008) realizaram um programa com dois pais e sete mães, tendo como principal objetivo maximizar as habilidades sociais educativas parentais. Para avaliar o impacto do programa, utilizaram medidas pré e pós-intervenção que avaliaram o repertório de habilidades sociais (IHS - Del Prette) e o repertório de habilidades sociais educativas parentais (QHSE-P). No total, foram 22 sessões de intervenção e oito sessões de avaliação. Como principais resultados, verificou-se que a intervenção apresentou impacto positivo no repertório de habilidades sociais e educativas (considerando as habilidades de: elogiar familiares, reagir a elogios, recusar pedidos abusivos, manter conversação, evitar o uso de práticas punitivas e estabelecer limites de forma mais positiva).

Barros e Del Prette (2007) avaliaram os efeitos de um programa de treinamento de habilidades sociais para pais sobre o desempenho acadêmico de crianças. Participaram do estudo 15 pessoas, 12 mães e 3 pais (8 do grupo experimental e 7 do grupo controle) de crianças do segundo ao nono ano ensino fundamental que tinham queixas escolares. A intervenção ocorreu em 13 sessões de 120 minutos de duração cada. Os resultados mostraram que os pais do GE: (a) ampliaram o repertório de habilidades sociais, com novas aquisições; (b) melhoraram a frequência, funcionalidade e fluência das habilidades sociais disponíveis (como a expressão de carinho e atenção, uso de reforçamento positivo, redução de problemas e de punições); (c) mantiveram os ganhos obtidos pelo programa, assim como a generalização dos mesmos. As crianças, por sua vez, tiveram melhoras significativas no desempenho escolar, avaliado pelo Teste de Desempenho Escolar.

Apesar da importância dos programas de intervenção para a família, nota-se que a maioria deles é direcionada apenas à mãe, sendo escassas as intervenções em que os pais participam, ou mesmo aquelas direcionadas apenas para a figura paterna (Cia et al., 2006; Coley, 2001; Hill \& Taylor, 2004; Lamb, 1997; Taylor \& Daniel, 2000). Com base nos poucos trabalhos sobre programas de intervenção precoce que envolveram o pai, verifica-se que os pais que participaram destes programas apresentaram ganhos diversos com impacto positivo em toda a família, como maior participação nas atividades escolares, maior interação com os filhos, diminuição dos problemas conjugais e do estresse e melhora no comportamento e no desempenho acadêmico dos filhos (Duch, 2005; Fagan \& Iglesias, 1999).

Dada a importância potencial do envolvimento paterno para a maximização do desenvolvimento infantil e a escassez de estudos focalizando especificamente a participação do pai em programas de intervenção direcionados para a figura paterna, nota-se a necessidade de realizar um programa de intervenção para o pai de uma criança iniciando as atividades escolares. Sendo assim, este estudo teve como objetivo avaliar a eficácia de um programa de intervenção para pais sobre o envolvimento paterno.

\section{Método}

Participantes

Para separar os efeitos da intervenção de mudanças produzidas por demais variáveis (história,

Psicol. Argum. 2014 jan./mar., 32(76), 139-150 
maturação da criança, mudanças de atitudes do pai, da mãe e dos professores de acordo com a idade da criança), formaram-se três grupos: experimental 1 (GE1, formado por pais que participaram da intervenção), experimental 2 (GE2, formado por pais cujas esposas participaram da intervenção) e controle (GC, formado por pais que quiseram participar da intervenção, mas que, por causa dos horários, nem eles nem suas esposas conseguiram participar). 0 grupo controle, por uma questão ética, recebeu a intervenção (após passarem pela coleta de dados) que não foi avaliada neste estudo. Os três grupos foram constituídos sem a utilização de sorteios.

Foram utilizados como critérios para ser participante: a criança viver com o pai e a mãe (biológicos ou não); a criança estar alfabetizada (considerou-se alfabetizada a criança que apresentou o mínimo de 5 pontos em escrita e aritmética e 14 pontos em leitura no TDE); o pai estar empregado e se interessar em participar da intervenção.

\section{Pais e mães}

Esta fase do estudo contou com a participação de 97 pais e mães (29 do GE1, 34 do GE2 e 34 do GC), de crianças do segundo e terceiro ano do ensino fundamental. A média de idade dos pais era de 35 anos, variando entre 23 e 58 anos, e a média de idade das mães era de 32 anos, variando entre 20 e 55 anos. Em relação à classe socioeconômica, 7,1\% das famílias eram da classe D, 50,5\% eram da classe C, $35,4 \%$ eram da classe B2 e 7,1\% eram da classe B1 (segundo o critério brasileiro, que objetiva medir o poder aquisitivo do consumidor e classifica a população em cinco classes socioeconômicas). Quanto à renda familiar, $1 \%$ dos pais tinha uma renda menor do que um salário mínimo, 24,2\% tinham renda de um a dois salários mínimos, 59,6\% tinham renda de dois a quatro salários mínimos, e 15,2\% tinham renda de mais de quatro salários mínimos. Todos os pais e 97\% das mães exerciam algum tipo de atividade remunerada.

\section{Crianças}

Foram participantes desta fase do estudo 99 crianças (29 do GE1, 36 do GE2 e 34 do GC), com idade média de oito anos, variando entre seis e nove anos. Destas crianças, 49 eram do sexo masculino e 50 do sexo feminino, sendo que $21,2 \%$ estavam no segundo ano e $78,8 \%$ estavam no terceiro ano do ensino fundamental.

\section{Local da coleta de dados}

A coleta de dados dos participantes e a intervenção com os pais ocorreram em duas escolas municipais e em uma escola estadual, localizadas em um município no interior do estado de São Paulo.

\section{Medidas avaliativas}

Foi utilizada a Avaliação do relacionamento pai-filho (Cia, 2005). Duas escalas tipo Likert compõem o instrumento e este avalia o envolvimento dos pais com seus filhos (versão paterna, materna e da criança): (a) "Escala de comunicação (verbal e não verbal) entre pai e filho": uma escala tipo Likert de 22 itens, com a pontuação dos itens variando entre 0 (nunca) e 365 (uma vez por dia) ( $\alpha=0,95$ para a escala avaliada pelo pai; $\alpha=0,96$ para a escala avaliada pela mãe e pela criança); (b) "Escala de participação do pai nas atividades escolares, culturais e de lazer do filho": uma escala tipo Likert de 19 itens, com a mesma forma de pontuação dos itens $(\alpha=0,96$ para a escala avaliada pelo pai e pela criança; $\alpha=0,95$ para a escala avaliada pela mãe).

Procedimento de coleta de dados

Para medir os impactos do programa de intervenção para pais sobre o envolvimento paterno e o desenvolvimento da criança, utilizou-se um delineamento pré e pós-teste com grupo controle não equivalente. Segundo Cozby (2006), nesse delineamento existem o grupo experimental e o grupo controle, mas os participantes não foram aleatoriamente selecionados, ou seja, foram distribuídos pelas condições. Apesar dos dois grupos poderem não ser equivalentes, os escores do pré-teste podem ser comparados com os escores do pós-teste.

Para que os pais e mães dessem seu consentimento para sua própria participação e a de seus filhos na pesquisa, foram entregues os Termos de Consentimento Livre e Esclarecido (TCLE). A coleta de dados seguiu o mesmo procedimento no pré-teste, no pós-teste e no follow-up (antes, imediatamente 
após e nove meses depois da intervenção com os pais e as mães, respectivamente).

Após o consentimento dos pais, o questionário "Avaliação do relacionamento entre pai e filho" foi aplicado com os pais e as mães, sob a forma de entrevista (tempo para aplicação de 20 minutos). Concomitante à coleta de dados com os pais, a pesquisadora entrou em contato com as crianças para explicar as atividades que estariam desenvolvendo e objetivando o estabelecimento do rapport. Em outro momento, foi aplicado o mesmo questionário nas crianças, mas sob a forma de entrevista (com tempo estimado de aplicação de 20 minutos).

\section{Intervenção}

A intervenção focou o aprimoramento do envolvimento paterno e o ensino de práticas parentais pró-acadêmicas que incentivem os filhos. Utilizaram-se técnicas cognitivo-comportamentais, tendo como objetivo orientar e trabalhar os participantes sobre: (a) os fundamentos da análise aplicada do comportamento; (b) a necessidade de motivar seus filhos a se comportar de maneira adequada e a ter comportamentos adequados aos estudos; (c) a identificação dos determinantes de comportamentos desadaptativos dos filhos; (d) a aplicação cotidiana dos procedimentos básicos de modificação do comportamento; (e) as crenças e valores dos pais. A intervenção se baseou no pressuposto de que as crianças precisam ser reforçadas de modo frequente, contingente, intenso, diferenciado e sistemático e precisam ter modelos adequados dos pais, apoiando-se em intervenções realizadas por Caballo e Simón (2005), Cooper e Cooper (2005), Del Prette e Del Prette (2005), Fagan e Iglesias (1999) e Pinheiro, Haase, Del Prette, Amarante e Del Prette (2006), entre outros teóricos que seguem uma abordagem cognitivo-comportamental.

Realizou-se a intervenção com os pais em um período de três meses (12 sessões), com encontros semanais de 90 a 120 minutos de duração. Em todas as sessões foi solicitada tarefa em casa (exceto na última sessão), sendo retomada e discutida na próxima sessão. Além disso, durante as sessões eram anotados os exemplos, comentários e relatos de tarefa de casa dos pais. As anotações eram realizadas ao longo da intervenção, para garantir a maior fidedignidade das mesmas.

Ao final de cada sessão, os pais responderam a um pequeno questionário para avaliar a sessão e foram entregues materiais explicativos (folders, folhetos, exemplos ilustrativos, entre outros) para que levassem para casa e pudessem consultar, quando necessitassem. Ao longo da intervenção, o desempenho dos pais foi avaliado por meio de tarefas em casa, nas quais estes descreveram as interações semanais com os filhos, a fim de avaliar a generalização da intervenção para o ambiente familiar (Caballo \& Simón, 2005; Del Prette \& Del Prette, 2005). A participação dos pais nas sessões foi, em média, 94,6\% (entre 90,5 e 100\%).

Durante o programa de intervenção, os pais poderiam solicitar atendimentos individuais com a pesquisadora em caso de dificuldades que não poderiam ser solucionadas durante os encontros. Além disso, foi estabelecido com os pais que haveria reposição das sessões para aqueles que precisassem faltar, antes de ocorrer a próxima sessão (porque havia vários grupos paralelos na mesma escola). Ao final de cada sessão, dois pais, em média, recebiam atendimento individual. No total, foram oferecidos oito grupos de pais.

\section{Follow-up}

A coleta de follow-up deu início após um ano da coleta de dados do pré-teste. Houve a desistência de 17 pais, mães e crianças (sendo dois da primeira escola, cinco da segunda escola e dez da terceira escola). Os motivos das desistências foram mudanças da família para fora da cidade e de instituição de ensino dos filhos.

\section{Procedimento de análise de dados}

Com o questionário respondido pelos pais, mães e crianças, foram realizadas análises de medidas de tendência central e dispersão. Para comparar os dados obtidos, entre os três grupos (GE1, GE2 e GC), no pré-teste, pós-teste e follow-up, utilizou-se o AVONA, MANOVA (usando o software SPSS para Windows). Para comparar os dados do pós-teste e follow-up foram considerados apenas os participantes que permaneceram na fase do follow-up.

Psicol. Argum. 2014 jan./mar., 32(76), 139-150 


\section{Resultados}

Realizou-se testes de chi-quadrado e Anova para averiguar diferenças entre os três grupos de participantes quanto aos dados sociodemográficos, e não foram encontradas diferenças estatisticamente significativas. Para avaliar o impacto do programa de intervenção, a curto e a longo prazo, sobre o envolvimento paterno, comparou-se: (a) as escalas de comunicação (verbal e não verbal) entre pai e filho; (b) a participação do pai nas atividades escolares, culturais e de lazer do filho, primeiramente, no pré-teste e no pós-teste e, em seguida, no pós-teste e no follow-up, entre o grupo experimental 1 , experimental 2 e controle (Tabela 1).

Por meio do teste de Anova, verificou-se que na fase de pré-teste, independentemente do informante, não houve diferenças estatisticamente significativas na frequência de comunicação entre pai e filho, comparando os três grupos. No entanto, na fase de

Tabela 1 - Escores nas medidas do envolvimento paterno, no pré-teste e no pós-teste: Comparação do GEl, GE2 e GC

\begin{tabular}{|c|c|c|c|c|c|c|c|c|c|c|}
\hline & \multicolumn{4}{|c|}{$\begin{array}{l}\text { Experimental } 1 \\
(\mathrm{~N}=29)\end{array}$} & \multicolumn{4}{|c|}{$\begin{array}{c}\text { Experimental } 2 \\
\quad(N=36)\end{array}$} & \multicolumn{2}{|c|}{ Controle } \\
\hline & \multirow{2}{*}{$\begin{array}{c}\begin{array}{c}\text { Pré- } \\
\text {-teste }\end{array} \\
\text { Média/ } \\
\text { D.P. }\end{array}$} & \multirow{2}{*}{$\begin{array}{c}\text { Pós- } \\
\text {-teste } \\
\text { Média/ } \\
\text { D.P. }\end{array}$} & \multicolumn{2}{|c|}{ Manova } & \multirow{2}{*}{$\begin{array}{c}\text { Pré-teste } \\
\text { Média } \\
\text { /D.P. }\end{array}$} & \multirow{2}{*}{$\begin{array}{c}\text { Pós-teste } \\
\text { Média } \\
\text { /D.P. }\end{array}$} & \multicolumn{2}{|c|}{ Manova } & \multirow{2}{*}{$\begin{array}{c}\begin{array}{c}\text { Pré- } \\
\text {-teste }\end{array} \\
\text { Média } \\
\text { /D.P. }\end{array}$} & \multirow{2}{*}{$\begin{array}{c}\begin{array}{c}\text { Pós- } \\
\text {-teste }\end{array} \\
\begin{array}{c}\text { Média } \\
\text { /D.P. }\end{array}\end{array}$} \\
\hline & & & $\mathbf{F}$ & gl & & & $\mathbf{F}$ & gl & & \\
\hline \multicolumn{11}{|l|}{ Avaliador - Pai } \\
\hline $\begin{array}{l}\text { Comunicação } \\
\text { entre pai e } \\
\text { filho. }\end{array}$ & $\begin{array}{l}147,1 \\
(98,2)\end{array}$ & $\begin{array}{c}231,9 \\
(123,9)\end{array}$ & $7,59 * *$ & $1 ; 28$ & $\begin{array}{l}137,3 \\
(82,7)\end{array}$ & $\begin{array}{c}188,6 \\
(110,8)\end{array}$ & $6,23^{*}$ & $1 ; 35$ & $\begin{array}{l}126,1 \\
(84,0)\end{array}$ & $\begin{array}{c}132,4 \\
(109,1)\end{array}$ \\
\hline $\begin{array}{l}\text { Participação do } \\
\text { pai nas ativida- } \\
\text { des escolares, } \\
\text { culturais e de } \\
\text { lazer do filho. }\end{array}$ & $\begin{array}{c}131,5 \\
(109,4)\end{array}$ & $\begin{array}{l}209,4 \\
(53,6)\end{array}$ & $10,2^{* *}$ & $1 ; 28$ & $\begin{array}{l}130,5 \\
(88,1)\end{array}$ & $\begin{array}{l}175,7 \\
(41,2)\end{array}$ & $7,93^{* *}$ & $1 ; 35$ & $\begin{array}{l}103,9 \\
(86,3)\end{array}$ & $\begin{array}{l}125,9 \\
(60,1)\end{array}$ \\
\hline \multicolumn{11}{|l|}{ Avaliador - Mãe } \\
\hline $\begin{array}{l}\text { Comunicação } \\
\text { entre pai e } \\
\text { filho. }\end{array}$ & $\begin{array}{c}155,6 \\
(104,3)\end{array}$ & $\begin{array}{c}234,2 \\
(120,0)\end{array}$ & $6,94^{*}$ & $1 ; 28$ & $\begin{array}{l}160,4 \\
(88,7)\end{array}$ & $\begin{array}{c}206,5 \\
(102,7)\end{array}$ & $5,29 *$ & $1 ; 35$ & $\begin{array}{l}119,5 \\
(92,2)\end{array}$ & $\begin{array}{c}135,9 \\
(112,4)\end{array}$ \\
\hline $\begin{array}{l}\text { Participação do } \\
\text { pai nas ativida- } \\
\text { des escolares, } \\
\text { culturais e de } \\
\text { lazer do filho. }\end{array}$ & $\begin{array}{c}136,5 \\
(106,8)\end{array}$ & $\begin{array}{l}224,3 \\
(72,6)\end{array}$ & $10,9 * *$ & $1 ; 28$ & $\begin{array}{l}123,2 \\
(80,7)\end{array}$ & $\begin{array}{l}198,7 \\
(41,3)\end{array}$ & $27,2^{* * *}$ & $1 ; 35$ & $\begin{array}{l}103,7 \\
(83,8)\end{array}$ & $\begin{array}{l}133,7 \\
(43,3)\end{array}$ \\
\hline \multicolumn{11}{|l|}{$\begin{array}{l}\text { Avaliador - } \\
\text { Filho }\end{array}$} \\
\hline $\begin{array}{l}\text { Comunicação } \\
\text { entre pai e } \\
\text { filho. }\end{array}$ & $\begin{array}{l}124,7 \\
(95,4)\end{array}$ & $\begin{array}{c}199,3 \\
(122,1)\end{array}$ & $6,58^{*}$ & $1 ; 28$ & $\begin{array}{l}131,1 \\
(81,3)\end{array}$ & $\begin{array}{c}196,6 \\
(112,0)\end{array}$ & $8,09^{* *}$ & $1 ; 35$ & $\begin{array}{l}106,9 \\
(74,6)\end{array}$ & $\begin{array}{c}137,2 \\
(116,7)\end{array}$ \\
\hline $\begin{array}{l}\text { Participação do } \\
\text { pai nas ativida- } \\
\text { des escolares, } \\
\text { culturais e de } \\
\text { lazer do filho. }\end{array}$ & $\begin{array}{l}110,8 \\
(90,0)\end{array}$ & $\begin{array}{l}154,0 \\
(47,2)\end{array}$ & $23,2^{* * *}$ & $1 ; 28$ & $\begin{array}{l}109,4 \\
(70,9)\end{array}$ & $\begin{array}{l}160,3 \\
(41,8)\end{array}$ & $40,5^{* * *}$ & $1 ; 35$ & $\begin{array}{c}98,5 \\
(77,4)\end{array}$ & $\begin{array}{l}104,3 \\
(41,5)\end{array}$ \\
\hline
\end{tabular}

\section{Legenda:}

${ }^{*} \mathrm{p}<0,05$;

${ }^{* *} \mathrm{p}<0,01$;

$* * * \mathrm{p}<0,001$. 
pós-teste, os pais do GE1 apontaram uma frequência de comunicação com o filho significativamente maior quando comparados com os pais do GC $(F(2 ; 96)=6,04, p<0,01)$, e as mães do GE1 e do GE2 apontaram uma frequência estatisticamente maior de comunicação entre pai e filho quando comparadas com as mães do GC $(F(2 ; 96)=6,69, \mathrm{p}<0,01)$.

Comparando os três grupos na fase de pré-teste, não houve diferenças estatisticamente significativas na frequência de participação do pai nas atividades escolares, culturais de lazer do filho, independentemente do informante. No entanto, na fase de pós-teste, os pais do GE1 apontaram uma frequência de participação nas atividades escolares, culturais e de lazer do filho significativamente maior quando comparados com os pais do GE2, que apontaram uma frequência estatisticamente maior de participação quando comparados com os pais do GC $(\mathrm{F}(2 ; 96)=$ $20,8 ; \mathrm{p}<0,001)$. Ainda comparando os três grupos, na fase de pós-teste, segundo a opinião das mães e dos filhos, os pais do GE1 e do GE2 apresentaram uma frequência estatisticamente maior do que os pais do GC $(F(2 ; 96)=25,2, p<0,001-$ mãe; $F(2 ; 96)$ $=16,9, \mathrm{p}<0,001-$ filho) na frequência de participação nas atividades escolares, culturais e de lazer dos filhos.

Verifica-se na Tabela 1 que os pais, mães e filhos do GE1 (F(104330,2; 28) = 7,59, p<0,01 - pai; $\mathrm{F}(89641,9 ; 28)=6,94, \mathrm{p}<0,01-$ mãe; $\mathrm{F}(80712,4$; 28) $=6,58, \mathrm{p}<0,05$ - filho) e do GE2 (F(47380,7; 35) $=6,33, \mathrm{p}<0,05-$ pai; $F(38341,4 ; 35)=5,29, \mathrm{p}<0,05$ - mãe; $F(77272,2 ; 35)=8,09, p<0,01$ - filho) apontaram uma frequência significativamente maior na comunicação entre pai e filho na fase do pós-teste.

Os pais, mães e filhos do GE1 (F(87959,6; 28= 10,2, p<0,01- pai; $F(111977,9 ; 28)=10,9, p<0,01$ - mãe; $F(31043,8 ; 28)=23,2, p<0,001$ - filho) e do GE2 $(F(36701,5 ; 35)=7,93, p<0,01-$ pai; $\mathrm{F}(102759,5 ; 35)=27,2, \mathrm{p}<0,001$ - mãe; $\mathrm{F}(47302,4$; $35)=40,5, p<0,001$ - filho) apontaram uma frequência significativamente maior de participação dos pais nas atividades escolares, culturais e de lazer dos filhos na fase do pós-teste (Tabela 2)

Comparando os três grupos por meio do teste de Anova, na fase de follow-up os pais do GE1 apontaram apresentar uma frequência de comunicação entre pai e filho e de participação nas atividades escolares, culturais e de lazer do filho significativamente maior quando comparados aos pais do GE2, que apontaram uma frequência estatisticamente maior de participação nas atividades escolares, culturais e de lazer dos filhos quando comparados com os pais do GC $(\mathrm{F}(2 ; 80)=57,0, \mathrm{p}<0,001 ; \mathrm{F}(2 ; 80)=16,4$, $\mathrm{p}<0,001$, respectivamente). Além disso, segundo a opinião das mães e das crianças, os pais do GE1 e do GE2 apresentaram uma frequência estatisticamente maior do que os pais do GC na frequência de comunicação entre pai e filho $(F(2 ; 80)=29,9, p<0,001$ - mãe; $F(2 ; 80)=11,6, p<0,001$ - filho) e de participação do pai nas atividades escolares, culturais e de lazer do filho $(F(2 ; 80)=13,4, p<0,001-$ mãe; $\mathrm{F}(2 ; 80)=7,8, \mathrm{p}<0,01$ - filho $)$.

Como mostra a Tabela 2, apenas na escala de comunicação entre pai e filho os pais do GE1 $(\mathrm{F}(29351,3 ; 23)=4,01, \mathrm{p}<0,1)$ e do GE2 $(\mathrm{F}(19967,1$; 30 ) $=3,90, p<0,1$ ) avaliaram uma frequência média estatisticamente maior na fase de follow-up quando comparada à fase de pós-teste. Nas demais escalas e com os demais avaliadores, não houve alterações significativas na frequência média entre as fases de pós-teste e de follow-up.

\section{Discussão}

Neste estudo, o contexto de desenvolvimento que foi alvo de investigação e modificação foi o ambiente familiar, por considerar a família como principal mantenedora e/ou modificadora de comportamentos infantis (Aunola \& Nurmi, 2005; Caballo \& Simón, 2005; Del Prette \& Del Prette, 2005; Pacheco, Alvarenga, Reppold, Piccinini, \& Hutz, 2005).

A escolha em desenvolver um programa de intervenção para pais foi baseada no pressuposto de que o desenvolvimento ocorre na interação entre um organismo com características próprias e as experiências que este acumula nos ambientes em que transita, por meio de processos proximais (Bronfenbrenner, 1999). Considerando que estes envolvem interações com pessoas, objetos e símbolos, sendo de natureza bidirecional e ocorrendo em ambientes imediatos, os pais tornam-se os principais responsáveis pelos conteúdos de uma parte significativa dos processos experienciados pela criança jovem e, além disso, podem ter uma influência significativa sobre as experiências da criança em ambientes em que não estejam presentes, como a escola.

Analisando as escalas usadas para avaliar o envolvimento paterno, notou-se que, de modo geral,

Psicol. Argum. 2014 jan./mar., 32(76), 139-150 
Tabela 2 - Escores nas medidas do envolvimento paterno, no pós-teste e no follow-up: Comparação do GEl, GE2 e GC

\begin{tabular}{|c|c|c|c|c|c|c|c|c|c|c|}
\hline & \multicolumn{4}{|c|}{$\begin{array}{l}\text { Experimental } 1 \\
(\mathrm{~N}=24)\end{array}$} & \multicolumn{4}{|c|}{$\begin{array}{l}\text { Experimental } 2 \\
(\mathrm{~N}=31)\end{array}$} & \multicolumn{2}{|c|}{ Controle } \\
\hline & \multirow{2}{*}{$\begin{array}{l}\begin{array}{l}\text { Pós- } \\
\text {-teste }\end{array} \\
\text { Média/ } \\
\text { D.P. }\end{array}$} & \multirow{2}{*}{$\begin{array}{l}\text { Follow- } \\
\text {-up }\end{array}$} & \multicolumn{2}{|c|}{ Manova } & \multirow{2}{*}{$\begin{array}{l}\begin{array}{l}\text { Pós- } \\
\text {-teste }\end{array} \\
\begin{array}{l}\text { Média } \\
\text { /D.P. }\end{array}\end{array}$} & \multirow{2}{*}{$\begin{array}{l}\begin{array}{l}\text { Follow- } \\
\text {-up }\end{array} \\
\text { Média } \\
\text { /D.P. }\end{array}$} & \multicolumn{2}{|c|}{ Manova } & \multirow{2}{*}{$\begin{array}{l}\begin{array}{l}\text { Pós- } \\
\text {-teste }\end{array} \\
\begin{array}{l}\text { Média } \\
\text { /D.P. }\end{array}\end{array}$} & \multirow{2}{*}{$\begin{array}{l}\text { Follow- } \\
\text {-up }\end{array}$} \\
\hline & & & $\mathbf{F}$ & gl & & & $\mathbf{F}$ & gl & & \\
\hline \multicolumn{11}{|l|}{ Avaliador - Pai } \\
\hline $\begin{array}{l}\text { Comunicação } \\
\text { entre pai e filho. }\end{array}$ & $\begin{array}{l}239,6 \\
(114,8)\end{array}$ & $\begin{array}{l}289,1 \\
(45,6)\end{array}$ & $4,01+$ & $1 ; 23$ & $\begin{array}{l}204,4 \\
(110,9)\end{array}$ & $\begin{array}{l}240,3 \\
(37,5)\end{array}$ & $3,90+$ & $1 ; 30$ & $\begin{array}{l}136,4 \\
(109,9)\end{array}$ & $\begin{array}{l}163,5 \\
(45,1)\end{array}$ \\
\hline $\begin{array}{l}\text { Participação do } \\
\text { pai nas atividades } \\
\text { escolares, culturais } \\
\text { e de lazer do filho. }\end{array}$ & $\begin{array}{l}215,9 \\
(51,5)\end{array}$ & $\begin{array}{l}218,3 \\
(46,3)\end{array}$ & ns & ns & $\begin{array}{l}178,1 \\
(38,0)\end{array}$ & $\begin{array}{l}181,3 \\
(53,9)\end{array}$ & ns & ns & $\begin{array}{l}133,9 \\
(55,2)\end{array}$ & $\begin{array}{l}141,6 \\
(41,8)\end{array}$ \\
\hline \multicolumn{11}{|l|}{ Avaliador - Mãe } \\
\hline $\begin{array}{l}\text { Comunicação } \\
\text { entre pai e filho. }\end{array}$ & $\begin{array}{l}244,6 \\
(110,4)\end{array}$ & $\begin{array}{l}247,6 \\
(43,2)\end{array}$ & ns & ns & $\begin{array}{l}216,3 \\
(106,3)\end{array}$ & $\begin{array}{l}218,5 \\
(52,1)\end{array}$ & ns & ns & $\begin{array}{l}142,5 \\
(113,3)\end{array}$ & $\begin{array}{l}142,8 \\
(54,8)\end{array}$ \\
\hline $\begin{array}{l}\text { Participação do } \\
\text { pai nas atividades } \\
\text { escolares, culturais } \\
\text { e de lazer do filho. }\end{array}$ & $\begin{array}{l}227,2 \\
(63,3)\end{array}$ & $\begin{array}{l}231,6 \\
(56,5)\end{array}$ & ns & ns & $\begin{array}{l}204,1 \\
(38,9)\end{array}$ & $\begin{array}{l}211,6 \\
(39,5)\end{array}$ & ns & ns & $\begin{array}{l}135,2 \\
(45,8)\end{array}$ & $\begin{array}{l}156,4 \\
(66,2)\end{array}$ \\
\hline Avaliador - Filho & & & & & & & & & & \\
\hline $\begin{array}{l}\text { Comunicação } \\
\text { entre pai e filho. }\end{array}$ & $\begin{array}{l}209,6 \\
(112,2)\end{array}$ & $\begin{array}{l}240,9 \\
(42,3)\end{array}$ & ns & ns & $\begin{array}{l}212,8 \\
(111,2)\end{array}$ & $\begin{array}{l}220,5 \\
(34,4)\end{array}$ & ns & ns & $\begin{array}{l}141,8 \\
(118,2)\end{array}$ & $\begin{array}{l}182,9 \\
(54,3)\end{array}$ \\
\hline $\begin{array}{l}\text { Participação do } \\
\text { pai nas atividades } \\
\text { escolares, culturais } \\
\text { e de lazer do filho. }\end{array}$ & $\begin{array}{l}161,8 \\
(74,9)\end{array}$ & $\begin{array}{l}186,7 \\
(47,4)\end{array}$ & ns & ns & $\begin{array}{l}153,9 \\
(95,3)\end{array}$ & $\begin{array}{l}169,2 \\
(42,7)\end{array}$ & ns & ns & $\begin{array}{l}108,9 \\
(79,8)\end{array}$ & $\begin{array}{l}136,1 \\
(50,6)\end{array}$ \\
\hline
\end{tabular}

Legenda:

$+\mathrm{p}<0,1$;

ns = não apresenta diferenças estatisticamente significativas.

Nota: Variação da pontuação $=0$ (nunca) a 365 (todos os dias).

não houve diferenças significativas entre os três grupos quanto à frequência do envolvimento paterno, ou seja, os grupos não apresentaram diferenças estatisticamente significativas nas médias de comunicação com os filhos e participação nas atividades escolares, culturais e de lazer dos filhos na primeira avaliação. Um envolvimento significativo com os filhos é preditor de maior satisfação do pai quanto a seu papel familiar, agindo diretamente na dinâmica familiar, pois aumenta a qualidade do relacionamento com a esposa (Carlson, 2006; Coley, 2001; Lamb, 1997; Matta \& Knudson-Martin, 2006).

Essas formas de envolvimento podem reverter em uma boa qualidade de interação com os filhos, favorecendo o desenvolvimento infantil, principalmente no que diz respeito aos aspectos socioemocionais e ao desempenho acadêmico. A interação positiva entre pai e filho é incompatível com práticas coercitivas nocivas para o desenvolvimento infantil (Brancalhone, Fogo \& Williams, 2004; Gomide, 2003). Essa interação positiva é caracterizada como um fator de proteção para o desenvolvimento da criança (Moreno, 2004). De fato, vários estudos mostraram uma relação positiva entre envolvimento paterno e o desempenho acadêmico, o autoconceito e o repertório de habilidades sociais das crianças, assim como uma relação negativa entre o envolvimento paterno e os problemas de comportamento apresentados pelas crianças (Atzaba-Poria, Pike \& Deater-Deckard, 2004; Cia \& Barham, 2005; Cia et al., 2006; Davidov \& Grusec, 2006; Duch, 2005; Englund, Luckner, Whaley \& Egeland, 2004; Fletcher, Steinberg \& WilliamsWheeler, 2004; Hill \& Taylor, 2004; Hong \& Ho, 
2005; Overstreet, Devine, Bevans \& Efreom, 2005; Scaramella \& Conger, 2004).

Considerando uma análise ao nível mesossistêmico (Bronfenbrenner, 1996), o que pode contribuir para esse alto envolvimento dos pais na educação dos filhos é a escola onde as crianças estudam, pois as três instituições de ensino valorizavam a participação dos pais nas atividades acadêmicas dos filhos (oferecendo horários flexíveis de reuniões escolares e outras atividades para os familiares de seu alunado). Essa alta valorização da escola quanto à participação dos pais nas atividades escolares das crianças, fica evidente pela postura das diretoras, que apresentaram interesse em seu desenvolvimento e se mostraram dispostas a auxiliar neste estudo.

Quanto à comunicação, nota-se que os pais apresentaram alta frequência de diálogo com o filho nas três fases de coleta de dados. 0 envolvimento na educação dos filhos torna-se mais importante diante das demandas específicas da fase de transição em que as crianças desta amostra se encontravam (início do ensino fundamental). Nessa fase, o desempenho acadêmico, o ajustamento ao ambiente escolar, o relacionamento com os companheiros, a adesão às regras de comportamento moral e o comportamento socialmente habilidoso constituem as principais tarefas adaptativas de desenvolvimento e requerem muitas e diversificadas habilidades da criança (Marturano, 2008), sendo fundamental a assistência dos pais nesse processo.

Em relação à participação dos pais nas atividades escolares dos filhos, comportamentos como incentivar os filhos a assumir responsabilidades por tarefas escolares, valorizar as conquistas acadêmicas, auxiliar os filhos nas lições de casa e acompanhar o progresso escolar dos filhos, participando e se interessando pelas atividades dos filhos, têm sido identificados como preditores do sucesso escolar por parte dos filhos (Cia, Pamplin \& Williams, 2008; Soares, Souza \& Marinho, 2004) e motivadores dos filhos para com os estudos.

Após o programa de intervenção com os pais, notou-se uma maior frequência de envolvimento paterno na comunicação entre pai e filho e na participação nas atividades escolares, culturais e de lazer do filho, na fase de pós-teste, segundo a opinião dos pais, mães e crianças. Como se trata da avaliação das mesmas medidas, por três informantes, são indicativos da fidedignidade dos dados coletados (Cozby, 2006).

Essas melhoras se mantiveram na fase de follow-up, sendo que a frequência de comunicação entre pai e filho aumentou, demonstrando ganhos do grupo de intervenção em longo prazo. Para que houvesse mais comunicação com os filhos, os pais precisariam se interessar mais nas atividades que os filhos realizavam, além de criar estratégias para aumentar o vínculo e a confiança na relação, aspectos que necessitavam de tempo e vontade de ambas as partes. Provavelmente, a intervenção mostrou-se eficaz para aumentar a frequência de interações intrafamiliares a nível microssistêmico.

A melhora significativa do envolvimento parental também foi apontada em outros programas de intervenção que trabalharam com pais e/ou mães (Duch, 2005; Fagan \& Iglesias, 1999; Freitas, 2005; Pinheiro et al., 2006). No entanto, ressalta-se que mesmo nos casos em que os pais não tenham participado diretamente da intervenção (GE2), a participação das esposas alcançou resultados positivos, transmitindo aos pais comportamentos mais adequados de envolvimento e a importância dos mesmos para o desenvolvimento dos filhos. Essa aprendizagem de esposa-marido pode ter ocorrido por modelação ou por instrução. É interessante ressaltar que, apesar de os pais e as mães dessa amostra não terem queixa clínica de problemas comportamentais e de desempenho acadêmico dos filhos e de terem um envolvimento frequente estes, o grupo (que teve um caráter preventivo) trouxe benefícios para a maximização da qualidade da interação entre pais e filhos, como apontado no estudo de Bolsoni-Silva et al. (2008).

Segundo Silva (2007), o "bom pai" é caracterizado não por ser o provedor financeiro da família, mas por se envolver em atividades familiares, incluindo cuidados com os filhos e ajuda nas tarefas de casa, sendo que esses pais estão se preocupando mais do que os pais das gerações anteriores com o bem-estar pessoal e material dos filhos e com o provimento de atividades culturais. Além disso, o papel dos pais torna-se mais importante quando os filhos estão em fases de transição do desenvolvimento, como ocorre com as crianças dessa amostra. Esse papel deve estar relacionado, além da disponibilidade aos filhos, ao apoio e envolvimento ativo na negociação da progressiva independência do filho, antecipando momentos onde tais recursos relacionados

Psicol. Argum. 2014 jan./mar., 32(76), 139-150 
à independência devem estar mais disponíveis. Os pais devem discutir com os filhos suas práticas e valores, negociando as regras estabelecidas. Além disso, devem monitorar os comportamentos dos filhos, prevendo possíveis intercorrências em seu desenvolvimento (Oliveira \& Costa, 2005).

Por fim, ressalta-se a alta participação dos pais no programa de intervenção. A intervenção ter sido baseada nas necessidades dos pais e a possibilidade de repor as sessões e de receber orientação individual podem ter contribuído para a alta participação dos pais. Além disso, todas as sessões eram avaliadas pelos pais, e por meio do relato das tarefas de casa foi possível adequar os procedimentos adotados em cada sessão de acordo com as necessidades de cada grupo.

\section{Considerações finais}

Em curto prazo e longo prazo, o programa de intervenção para pais mostrou-se eficaz em termos de impacto sobre o aumento da frequência de envolvimento paterno, segundo os pais, as mães e as crianças.

Destaca-se a importância deste estudo ter sido conduzido em três instituições de ensino com diferentes populações, contribuindo para a confiança na generalização dos resultados, pelo menos na região do Brasil no qual ele foi conduzido. No entanto, os resultados envolvem dados obtidos com base em relatos dos pais e mães, o que pode sofrer diversos tipos de vieses. Estudos futuros poderiam complementar e ampliar a validade desses achados, incluindo dados de observação direta, tanto do repertório dos pais como dos filhos. Além disso, o instrumento "Avaliação do relacionamento entre pai e filho" não está validado no contexto brasileiro, o que mostra a necessidade de mais estudos para aprimorá-lo. Para um tipo de validação, por exemplo, torna-se necessário que este seja aplicado com uma população maior e que passe por análises fatoriais.

Com estas ressalvas, acredita-se que este trabalho faz uma contribuição de fundamental importância para melhor entender formas de promover o desenvolvimento de crianças nas condições sociais brasileiras atuais. Perante as demandas sociais que permitem e requerem um maior envolvimento paterno para manter condições positivas no âmbito familiar para o bom desenvolvimento de crianças e para enriquecer a vida de todos que entram em contato com estas crianças, começando com os próprios pais, existe uma necessidade urgente de contar com estudos como este, que permite analisar de forma sistemática os impactos de esforços de intervenção, devidamente explicados e documentados.

\section{Referências}

Ahmed, S. S., \& Bould, S. (2004). One able daughter is worth 10 illiterate sons: Reframing the patriarchal family. Journal of Marriage and Family, 66, 1332-1341.

Atzaba-Poria, N., Pike, A., \& Deater-Deckard, K. D. (2004). Do risk factors for problem behavior act in a cumulative manner? An examination of ethnic minority and majority children through an ecological perspective. Journal of Child Psychology and Psychiatry, 45(4), 707-718.

Aunola, K., \& Nurmi, J. E. (2005). The role of parenting styles in children's problem behavior. Child Development, 76(6), 1144-1159.

Barros, S. K. S. N., \& Del Prette, A. (2007). Um treinamento de habilidades sociais para pais pode beneficiar os filhos na escola? Revista da Sociedade e Psicologia do Triângulo Mineiro, 11(1), 107-123.

Biasoli-Alves, Z. M. M., Caldana, L. H. R., \& Silva, F. G. M. D. (1997). Práticas de educação da criança na família: A emergência do saber técnico científico. Revista Brasileira Crescimento e Desenvolvimento Humano, 7(1), 49-62.

Bolsoni-Silva, A. T., Salina-Brandão, A., Rosin-Pinola, A. R., \& Versuti-Stoque, F. M. (2008). Avaliação de um programa de intervenção de habilidades sociais educativas parentais: Um estudo-piloto. Psicologia: Ciência e Profissão, 28(1), 18-33.

Brancalhone, P. G., Fogo, J. C., \& Williams, L. C. A. (2004). Crianças expostas à violência conjugal: Avaliação do desempenho acadêmico. Psicologia: Teoria $e$ Pesquisa, 20(2), 113-117.

Brandth, B., \& Kvande, E. (2002). Reflexive fathers: Negotiating parental leave and working life. Gender, Work and Organization, 9(2), 186-203. 
Bronfenbrenner, U. (1996). A ecologia do desenvolvimento humano: Experimentos naturais e planejados. Porto Alegre: Artes Médicas.

Bronfenbrenner, U. (1999). Environments in developmental perspective: Theoretical and operational models. Em Y. L. Fiedman, \& T. D. Wachs (Orgs.), Captation and assessment of environment across the life (pp. 3-30). Washington: American Psychological Association.

Caballo, V. E., \& Simón, M. A. (2005). Manual de psicologia clínica e do adolescente: Transtornos específicos. São Paulo: Santos.

Carlson, M. J. (2006). Family structure, father involvement and adolescence behavioral outcomes. Journal of Marriage and Family, 68, 137-154.

Cia, F. (2005). O impacto do turno de trabalho do pai no desempenho acadêmico e no autoconceito de crianças escolares. Dissertação de Mestrado, Programa de Pós-Graduação em Educação Especial, Universidade Federal de São Carlos, São Carlos.

Cia, F., \& Barham, E. J. (2005). A relação entre o turno de trabalho do pai e o autoconceito do filho. Psico, 36(1), 29-35.

Cia, F., Pamplin, R. C. O., \& Del Prette, Z. A. P. (2006). Comunicação e participação Pais-filhos: Correlação com habilidades sociais e problemas de comportamento dos filhos. Paideia, 16(35), 395-406.

Cia, F., Pamplin, R. C. O., \& Williams, L. C. A. (2008). O impacto do envolvimento parental no desempenho acadêmico de crianças escolares. Psicologia em Estudo, 13(2), 251-260.

Cia, F., Pereira, C. S., Del Prette, Z. A. P., \& Del Prette, A. (2006). Habilidades sociais parentais e o relacionamento entre pais e filhos. Psicologia em Estudo, 11(1), 73-81.

Coley, R. L. (2001). (In)visible Men: Emerging research on low-income, unmarried, and minority fathers. American Psychologist, 56(9), 743-753.

Cooper, B., \& Cooper, N. (2005). What a difference a parent makes! New York: A focus on father workbook to accompany.
Cozby, P.C. (2006). Métodos de pesquisa em ciências do comportamento. São Paulo: Atlas.

Dantas, C., Jablonski, B., \& Féres-Carneiro, T. (2004). Paternidade: Considerações sobre a relação pais-filhos após a separação conjugal. Cadernos de Psicologia e Educação Paidéia, 14(29), 347-357.

Davidov, M., \& Grusec, J. E. (2006). Untangling the links of parental responsiveness to distress and warmth to child outcomes. Child Development, 77(1), 44-58.

Del Prette, Z. A. P., \& Del Prette A. (2005). Psicologia das habilidades sociais na infância: Teoria e prática. Petrópolis: Vozes.

Duch, H. (2005). Redefining parent involvement in Head Start: A two-generation approach. Early Child Development and Care, 175(1), 23-35.

Englund, M. M., Luckner, A. E., Whaley, G. J. L., \& Egeland, B. (2004). Children's achievement in early elementary school: Longitudinal effects of parental involvement, expectations, and quality of assistance. Journal of Educational Psychology, 96(4), 723-730.

Fagan, J., \& Iglesias, A. (1999). Father involvement program effects on fathers, father figures, and their Head Start children: A quasi-experimental study. Early Childhood Research Quarterly, 14(2), 243-269.

Fleck, A., \& Wagner, A. (2003). A mulher como a principal provedora do sustento econômico familiar. Psicologia em Estudo, 8(número especial), 31-38.

Fletcher, A. C., Steinberg, L., \& Williams-Wheeler, M. (2004). Parental influences on adolescent problem behavior revisiting Stattin and Kerr. Child Development, 75(3), 781-796.

Flouri, E., \& Buchanan, A. (2003). The role of father involvement in children's later mental health. Journal of Adolescence, 26, 63-78.

Freitas, M. G. (2005). Desenvolvimento e avaliação de um programa de habilidades sociais com mães sobre o repertório social dos filhos deficientes visuais. Tese de Doutorado, Programa de Pós-graduação em Educação Especial, Universidade Federal de São Carlos, São Carlos.

Psicol. Argum. 2014 jan./mar., 32(76), 139-150 
Gomide, P. I. C. (2003). Estilos parentais e comportamento antissocial. Em A. Del Prette \& Z. A. P. Del Prette (Orgs.), Habilidades sociais, desenvolvimento e aprendizagem (pp. 21-60). Campinas: Alínea.

Guille, L. (2004). Men who better and their children: An integrated review. Aggression and Violent Behavior, 9, 129-163.

Hawkins, D. N., Amato, P. R., \& King, V. (2006). Parentadolescent involvement: The relative influence of parent gender and residence. Journal of Marriage and Family, 68, 125-136.

Hill, N. E., \& Taylor, L. C. (2004). Parental school involvement and children's academic achievement. Current Directions in Psychological Science, 13(4), 161-164.

Hong, S., \& Ho, H. (2005). Direct and indirect longitudinal effects of parental involvement on student achievement: Second-Orde latent growth modeling across ethnic groups. Journal of Educational Psychology, 97(1), 32-42.

Lamb, M.E. (1997). Fathers and child development: An introductory overview and guide. In M. E. Lamb (Org.), The role of the father in child developmental (pp. 1-18). New York: John Wiley \& Sons.

Marturano, E. M. (2008). Tensões cotidianas na transição da primeira série: Um enfoque de desenvolvimento. Psicologia em Estudo, 13(1), 79-87.

Matta, D. S., \& Knudson-Martin, C. (2006). Father responsivity: Couple processes and the co-construction of fatherhood. Family Process, 45(1), 19-37.

McMahon, R. J. (1999). Treinamento de pais. In V. E. Caballo (Org.), Manual de técnicas de terapia e modificação do comportamento (pp. 399-422). São Paulo: Santos.

Moreno, M. D. C. (2004). Desenvolvimento e conduta social dos seis anos até a adolescência. In C. Coll, A. Marchesi, \& J. Palácios (Orgs.), Desenvolvimento psicológico e educação - Psicologia evolutiva (pp. 287305). Porto Alegre: Artmed.

Oliveira, J., \& Costa, M. E. (2005). Estilos de vinculação e percepções de satisfação com os papéis parental e conjugal em tríades de famílias intactas. Psicologia, 18(2), 57-74.
Overstreet, S., Devine, J., Bevans, K., \& Efreom, Y. (2005). Predicting parental involvement in children's schooling within an economically disadvantaged African American sample. Psychology in the Schools, 42(1), 101-111.

Pacheco, J., Alvarenga, P., Reppold, C., Piccinini, C. A., \& Hutz, C. S. (2005). Estabilidade do comportamento antissocial na transição da infância para a adolescência: Uma perspectiva desenvolvimentista. Psicologia: Reflexão e Crítica, 18(1), 55-61.

Pinheiro, M. I. S., Haase, V. G., Del Prette, A., Amarante, C. L. D., \& Del Prette, Z. A. P. (2006). Treinamento de habilidades sociais educativas para pais de crianças com problemas de comportamento. Psicologia: Reflexão $e$ Crítica, 19(3), 1-15.

Raley, S. B., Mattlingly, M. J., \& Bianchei, S. M. (2006). How dual are dual-income couples? Documenting change from 1970 to 2001. Journal of Marriage and Family, $68,11-28$.

Rocha-Coutinho, M. L. (2003). Quando o executivo é uma "dama": A mulher, a carreira, e as relações familiares. In T. Féres-Carneiro (Org.), Família e casal: Arranjos e demandas contemporâneas (pp. 15-30). Rio de Janeiro: NAU.

Scaramella, L. V., \& Conger, R. D. (2004). Intergenerational continuity of hostile parenting and its consequences: The moderating influence of children's negative emotional reactivity. Social Development, 12(3), 420-439.

Silva, M. M. C. (2007). Vida profissional e familiar: Padrões de conflito e facilitação na gestão de múltiplos papéis. Dissertação de Mestrado, Faculdade de Psicologia e de Ciências da Educação, Universidade do Porto, Porto.

Soares, M. R. Z.; Souza, S. R., \& Marinho, M. L. (2004). Envolvimento com os pais: Incentivo à habilidade de estudo em crianças. Estudos de Psicologia, 21(3), 253-260.

Taylor, J., \& Daniel, B. (2000). The rhetoric versus the reality in childcare and protection ideology and practice, in working with fathers. Journal of Advanced Nursing, 31(1), 12-19. 